\title{
Autism Spectrum Disorder (ASD) in a Developing country. A Clinical and Descriptive Study of 75 Infant and Young Patients
}

\author{
Castejón 0J*, Galindez P, Torres IA, Villasmil A, Grudbasum E, Castejon EM and Salones \\ de Castejón M
}

Facultad de Medicina, Venezuela

*Corresponding author: Orlando J Castejón, Facultad de Medicina, Venezuela

\section{ARTICLE INFO}

Received: 㓞 July 01, 2019

Published: 蔧 July 11, 2019

Citation: Castejón OJ, Galindez P, Torres IA, Villasmil A, Grudbasum E, Castejon EM, Salones de Castejón M. Autism Spectrum Disorder (ASD) in a Developing country. A Clinical and Descriptive Study of 75 Infant and Young Patients. Biomed J Sci \& Tech Res 19(4)-2019. BJSTR. MS.ID.003321.

Abbreviations: ASD: Autism Spectrum Disorder; RRBs: Restricted, Repetitive Behaviors; MRI: Magnetic Resonance Imaging; ADHD: Attention Deficit Hyperactivity Disorder; EEG: Electroencephalography; IED: Interictal Epileptiform Discharges; APLS: Antiphospholipid Syndrome; TPO: Thyroid Peroxidase; BBB: Blood Brain Barrier; MCs: Mast Cells; CRF: Corticotropin Releasing Factor; NT: Neurotensin; FXS: Fragile X Syndrome; OCD: Obsessive Compulsive Disorders; PMS: Phelan McDermid Syndrome

\author{
ABSTRACT
}

Seventy five (75) infant patients, from 3-15 years old, clinically diagnosed with autism spectrum disorder exhibited disconnected brain with social isolation (80\%), hyperconnected children (5\%), creative child (Asperger syndrome) (10\%), stereotyped movements of hands and body trunk (10\%), hyperactivity and attention deficit $(10 \%)$, language disorders such as delay in the onset of language or early vocalization, regressive changes of language, mutism, gestual language, escatologic language, digital language (5\%), learning and memory deficit (10\%), psychomotor delay (5\%), behavioral changes, such as aggresivity and autoagressivity (10\%), tactile hypersensitivity, crisis of cry (1\%), separation anxiety (1\%), mood disorders such as anxiety and depression (3\%), photofobia $(1 \%)$, loss of weight, $(1 \%)$, routinised patterns of though and fantastic thoughts $(2 \%)$.

The following associated comorbidities: perinatal hypoxia, low weight at birth, behavioral abnormities, anxiety, auto- and heteroagressivity, hyperphagia, learning and hearing disorders, mainly hyperacusia, social isolation, cognitive deficit, sleeping disorders and parenteral abuse of child. Some non-nervous system comorbidities, such as allergic reaction, bronchial asthma and atopic dermatitis also were found. Some locomotor abnormalities as genus valgo and flat feet were also observed. The mothers exhibited some of the followings diseases during pregnancy: urinary infections, behavioral disturbances like anxiety, fobias, hyperactivity, toxoplasmosis and Zika virus infections, hyperemesis, oligohydramnios and lost of amniotic fluid, twin pregnancy, pre-eclampsia, aging placenta, cesarean surgery, high blood pressure, maternal sepsis, diabetes, hepatic coma, hypotiroidism, viral hepatitis, obesity, and social parenteral problems, such as excessive work, low economy and poor social conditions, environmental contamination and labor and conjugal stress.

Keywords: Autism; ASD Risk Factor; ASD Comorbidities

\section{Introduction}

Autism spectrum disorder (ASD) involves a complex interplay of both genetic and environmental risk factors, with immune alterations and synaptic connection deficiency in early life. In the past decade, studies of ASD have substantially increased, in both humans and animal models. Immunological imbalance (including autoimmunity) has been proposed as a major etiological component in ASD, taking into account increased levels of pro-inflammatory cytokines observed in postmortem brain from patients, as well as autoantibody production [1]. Evidence indicates an increased offspring vulnerability to ASD through advanced maternal and paternal age, valproate intake, toxic chemical exposure, maternal diabetes, enhanced steroidogenic activity, immune activation, and possibly altered zinc-copper cycles, and treatment with selective serotonin reuptake inhibitors [2]. The etiology of this disorder, however, is largely unknown, although the environmental relative to the genetic contribution is substantial [3].

The genetic architecture of ASD has become increasingly clear and increasingly complex with estimates of at least 1000 genetic alterations associated with the risk for ASD. A consensus has emerged that genetic and environmental causes of ASD are likely 
multifactorial [4]. An associations between early developmental exposure to ambient pesticides and autism spectrum disorder has been recently reported [5,6]. Etiology of ASD likely involves environmental factors triggering physiological abnormalities in genetically sensitive individuals. One of these major physiological abnormalities is mitochondrial dysfunction, which may affect a significant subset of children with ASD. Co-occurring medical disorders and associated physiological abnormalities in individuals with autism spectrum disorder (ASD) show strongest associations with epilepsy, sleep, as well as gastrointestinal and immune functioning [7]. Individuals with autism spectrum disorder (ASD) exhibit severe difficulties in social interaction, motor coordination, behavioral flexibility, and atypical sensory processing, with considerable interindividual variability [8].

Autism spectrum disorder (ASD) is a neurodevelopmental disorder that is clinically defined by abnormalities in reciprocal social and communicative behaviors and an inflexible adherence to routinised patterns of thought and behavior. Laboratory studies repeatedly demonstrate that autistic individuals experience difficulties in recognizing and understanding the emotional expressions of others and naturalistic observations show that they use such expressions infrequently and inappropriately to regulate social exchanges [9]. In a previous work we have reported the maternal stress and the relationship with hyperactivity and attention deficit (ADHD) in ASD patients [10]. In the present study we have carried out a clinical and descriptive study of autism spectrum disorder (ASD), and the involved risk factors and comorbidities in a developing country facing a severe and critical sociopolitical situation. We emphasize the maternal pathological processes during pregnancy, an underestimated pathology thus far as an essential risk factor for ASD, mainly in developing countries facing sociopolitical crisis.

\section{Material and Methods}

We have examined 75 infant patients ranging from 3 to 15 years-old years-old with autism spectrum disorder. The children were clinically studied at the Clinical Neuroscience Outpatient Clinic of Clinical Neuroscience Institute at Clinical Home San Rafael de Maracaibo. Venezuela. They were previously examined from the psychological point of view at CETRO, Maracaibo, or at different Public Psychology Centers of Maracaibo City. We have followed the criteria established in the Diagnostic and Statistical Manual of Mental Disorders (DSM-5) and the principles of Helsinki Declaration for Research in Human Beings.

\section{Results}

In the present clinical study on autism spectrum disorder we have examined 75 infant patients exhibiting disconnected child, prevalent in males than females, with social isolation (80\%), hyperconnected, and creative child (Asperger syndrome) $(10 \%)$, stereotypic movements of hands and body (15\%), tactile hypersensitivity and altered pain perception (10\%), hyperactivity and attention deficit (10\%), behavioral changes such as aggresivity and autoagressivity (10\%), learning and memory deficit $(10 \%)$, separation anxiety $(1 \%)$, mood disorders ( $3 \%)$, routinised patterns of though and fantastic thoughts (2\%), language disorders such as delay in the onset of language or early vocalization, regressive changes of language, mutism, gestual, escatologic and digital language (5\%), psycomotor delay (5\%) and gait disorder (3), crisis of cry (1\%), photofobia (1\%), loss of weight, (1\%), and gastrointestinal and hepatic diseases, such as esophageal atresia and gastric reflux, constipation or diarrhea, neonatal jaundice (3\%), cardiac malformation and intraventricular communication(1\%), hyperactivity and attention deficit (30\%), neurosensory disorders (2\%), such as hyperacusia o hypoacusia, visual defects as strabismus and myopia (1\%), hyperphagia (2\%), convulsive syndrome and febrile seizures (3\%), sleep disorders (5\%), TV, cellular phone, table and computer addiction (15\%), hypothiroidism, allergic diseases, such as bronchial asthma and atopic dermatitis (3\%). Some locomotor abnormalities like genus valgo and flat feet also were observed (2\%).

The following risks factors were found: advanced maternal and paternal age (4\%), prematurity (10\%), family background of autism (3\%), perinatal hypoxia $(10 \%)$, parenteral abuse of child (5\%). The mothers exhibited the followings diseases during pregnancy: urinary infections $(20 \%)$, behavioral disturbances like anxiety (10\%), fobias (1), hyperactivity and attention deficit (20\%), toxoplasma and Zika virus infections (2), hyperemesis (25), oligohydramnios and loss of amniotic fluid (2\%), twin pregnancy $(1 \%)$, pre-eclampsia $(3 \%)$, aging placenta $(2 \%)$, cesarean surgery ( $2 \%$ ), high blood pressure (1\%), maternal sepsis, diabetes and hepatic coma (1\%), hypotiroidism (1\%), viral hepatitis (1\%), parent obesity (2\%), and social problems, such as excessive work, low economy and poor conditions environmental contamination, labor and conjugal stress (10\%).

\section{Discussion}

\section{Stereotyped Movements of Hands and Body in ASD}

Fifteen per cent of patients examined in the present study showed stereotyped movements of hands and body. Stereotypical motor movements are one of the most common and least understood behaviors occurring in individuals with Autism Spectrum Disorder (ASD). Restricted, repetitive behaviors (RRBs) are patterns of behavior that exhibit little variation in form and have no obvious function and relatively little is known about the neural circuitry of RRBs. Alterations in frontostriatal circuitry might underlie this behavioral impairment. Magnetic resonance imaging (MRI) is wellsuited to studying the structural and functional connectivity of the nervous system and is a highly translational research tool. These studies implicate distributed neural circuits between the cortex, basal ganglia, and cerebellum [11] (Wilkes and Lewis, 2018). Cai et al. [12] have reported increased left inferior temporal gyrus in both low function autism and high function autism. 
Tactile Hypersensitivity and GABA Concentration in the Sensorimotor Cortex

In the present study we have found tactile hypersensitivity in a $10 \%$ of patients examined typically characterized by either over- or under-responsiveness to stimulation. Impaired responses to tactile stimulation are a commonly reported symptom among children with autism spectrum disorder (ASD) suggesting a functional deficit in the somatosensory inhibitory system in autism [13]. Puts et al. According to Puts et al. (2017) and Sapey-Triomphe et al. [14]. tactile hypersensitivity is correlated with reduced GABA levels measured by magnetic resonance spectroscopy in brain areas processing touch, such as sensorimotor cortex.

\section{Hyperconnected ASD Children (Asperger syndrome)}

In the present study we have found 10\% ASD children with higher functioning activity. Asperger's syndrome seems to be considerably more common than "classic" autism. The syndrome is more prevalent in boys than in girls. The clinical characteristics of Asperger's syndrome are probably influenced by many factors, including organic and genetic factors [15] . Compared with healthy controls, ASD children showed significantly increased functional connectivity between the left premotor area and the posterior cingulate gyrus or anterior lobe of wedge [16]. While predominant overconnectivity was found for posterior cingulate seed and right inferior parietal seed, predominant underconnectivity was found for right anterior insula seed and left inferior parietal seed [17]. According to Rinehart et al. [18] there is contemporary conceptualizations of autism and Asperger's disorder, together with epidemiological information, genetic and neurobehavioural findings on neurobehavioural studies, in particular, executive functioning, lateralization, visual-perceptual and motor processing, which have provided an important source of information about the potential neurobiological dissociation that may exist between autism and Asperger's disorder. On the contrary, according to Macintosh and Dissanayake [19] there is currently insufficient evidence to establish the validity of Asperger's disorder as a syndrome distinct from high-functioning autism. The findings are consistent with the view that these disorders belong on an autism spectrum. It is well known that the cases with savant syndrome, demonstrate outstanding mental capability despite coexisting severe mental disabilities. In many cases, savant skills are characterized by its domain-specificity, enhanced memory capability, and excessive focus on low-level perceptual processing In addition, impaired integrative cognitive processing such as social cognition or executive function, restricted interest, and compulsive repetition of the same act are observed in savant individuals. All these are significantly relevant to the behavioral characteristics observed in individuals with autistic spectrum disorders (ASD).

A neurocognitive model of savant syndrome should explain these cognitive features and the juxtaposition of outstanding talents with cognitive disabilities [20]. According to these Authors, the disruption of connectivity between the prefrontal cortex and other regions is considered to be a particularly important factor because the prefrontal region shows the most influential inhibitory control on other cortical areas. We propose that these neural mechanisms as the underlying causes for the emergence of savant ability in ASD.

\section{ASD and Psychomotor Delay}

We have found psychomotor delay in $5 \%$ of ASD patients. Altered motor performance has been described in Autism Spectrum Disorders (ASD) with disturbances in walking; posture, coordination, or arm movements, but some individuals with ASD show no impairment of motor skills. Paquet et al. [21,22] suggest a semiology of tone deregulation between proximal versus distal muscles, indeterminate tonic laterality, postural control deficit (proprioceptive), impairment of inter-hemispheric coordination (corpus callosum), and neurological soft signs such as dysdiadochokinesia, which leads us to hypothesize a general impairment of motor functions. Shevell et al. [23] earlier postulated four diagnoses (cerebral dysgenesis, hypoxic-ischemic encephalopathy, toxin exposure, chromosomal abnormalities) accounted for

global developmental delay.

In our studied we have found the followings diseases during mother pregnancy: urinary infections, behavioral disturbances like anxiety, fobias, hyperactivity, toxoplasmosis and Zika virus infections, viral hepatitis, viral hepatitis, viral hepatitis, hyperemesis, oligohydramnios and lost of amniotic fluid, twin pregnancy, pre-eclampsia, aging placenta, cesarean surgery, high blood pressure, maternal sepsis, diabetes, hepatic coma, hypothyroidism, obesity, and social parenteral problems, such as excessive work, low economy and poor social conditions, environmental contamination, labor and conjugal stress. Such maternal pathology could be considered as risk and/or etiological factors in ASD children. We should keep in mind that this maternal pathology is frequently observed in developing countries, mainly in those facing sociopolitical crisis.

\section{Language Disorders in ASD and Sensory Processes Alterations}

In the present study we have found language disorders in a $5 \%$ of patients studied, such as delay in the onset of language or early vocalization, regressive changes of language, mutism, gestural, escatologic and digital language. Sensory processing alterations are highly prevalent in autism spectrum disorder (ASD). Neurobiologically-based theories of ASD propose that abnormalities in the processing of temporal aspects of sensory input could underlie core symptoms of ASD. For example, rapid auditory temporal processing is critical for speech perception, and language difficulties are central to the social communication deficits defining the disorder. Results indicate a domain-specific impairment in rapid auditory temporal processing in ASD that is associated with greater difficulties in language processing [24]. 
There is significant clinical heterogeneity in language and communication abilities of individuals with autism spectrum disorders (ASD). However, no consistent pathology regarding the relationship of these abilities to brain structure has emerged [25]. Lo et al. [26] hypothesized that the dual stream language network is altered in autism, and that this alteration could be revealed by changes in the relationships between microstructural integrity and functional activation. Microstructural integrity of the left dorsal and left ventral pathways responsible for language processing and the functional activation of the connected brain regions were studied in youths with high-functioning autism. The altered structurefunction relationships in autism suggest possible involvement of the dual pathways in supporting deficient semantic processing.

\section{Sensory and Multisensory Function in Autism Spectrum Disorder}

We found in ASD children neurosensory disorders, such as hyperacusia o hypoacusia (2\%), visual defects as strabismus and myopia (1\%) in patients with ASD. These disorders are related with language disorders and learning and memory deficit found in ASD. Sensory problems are included as part of the core symptoms of autism spectrum disorder (ASD) in the diagnostic profile. Because sensory information forms the building blocks for higher-order social and cognitive functions, Baum et al. [27] argue that sensory processing is not only an additional piece of the puzzle, but rather a critical cornerstone for characterizing and understanding ASD. These Authors emphasized the importance of multisensory processing in building perceptual and cognitive representations, and how deficits in multisensory integration may also be a core characteristic of ASD. Increased pain sensitivity and increased touch sensitivity in areas classically related to affective touch (C-tactile afferents innervated areas) may explain typical avoiding behaviors associated with hypersensitivity [28]

Recent work suggests sensory seeking predicts later social symptomatology through reduced social orienting in infants who are at high-risk for autism spectrum disorder (ASD) [29]. Neurobiological research that has been conducted has pointed toward dysfunction in the excitation/inhibition balance of the central nervous system of those with ASD. Future endeavors into understanding tactile processing differences in ASD will greatly benefit from controlled experiments driven by neurobiological hypotheses [30]

\section{Learning and Memory Deficit in ASD}

We have found 10\% learning and memory deficit in the ASD patients examined. SHANK3 is a synaptic scaffolding protein localized in the postsynaptic density and has a crucial role in synaptogenesis and neural physiology. Deletions and point mutations in SHANK3 cause Phelan-McDermid Syndrome (PMS) and have also been implicated in autism spectrum disorder (ASD) and intellectual disabilities, leading to the hypothesis that reduced SHANK3 expression impairs basic brain functions that are important for social communication and cognition [31]. Contrary to expectations, children with ASD demonstrated a relative weakness in the rate of acquisition of visual in contrast to verbal learning compared to neurotypicals. They also showed a complex pattern of consolidation. Overall, between-group differences were more likely to emerge during the visual learning task, suggesting that it may be more sensitive for detecting neurodevelopmental differences [32].

Abnormal visual processing as well as auditory processing disorders eventually lead directly to learning disorders in children and young adults [33] Kantzer et al. [34] found borderline intellectual functioning, and intellectual developmental disorder in community pre-schoolers.

\section{Aggressivity and Autoagressivity in ASD}

In the present study we found $10 \%$ of ASD patients with aggressivity and autoagressivity. Aggression is common in patients with autism spectrum disorders (ASD) along with the core symptoms of impairments in social communication and repetitive behavior. Risperidone, an atypical antipsychotic, is widely used to treat aggression in ASD [35]. Both attention-deficit hyperactivity disorder (ADHD) and autism spectrum disorder (ASD) are frequently accompanied by serious aggression that requires psychiatric treatment [36]. Sener et al. [37] analyzed nine mRNA expressions of the candidate genes related to aggression and insensitivity to pain in the peripheral blood of patients with ASD. Alterations in the mRNA expression patterns of the HTR1E, OPRL1, OPRM1, TACR1, PRKG1, SCN9A and DRD4 genes provide further evidence for a relevant effect of the respective candidate genes on the pathophysiology of ASD.

\section{ASD and Sleep Disorders}

We have found sleep disorders in a 5\% of ASD patients. Liu et al. [38] examined sleep patterns, sleep problems, and their correlates in children with autism spectrum disorders, and found ASD patients with bedtime resistance, insomnia, parasomnias, sleep disordered breathing, morning rise problems, and with daytime sleepiness. Their results showed that both dyssomnias and parasomnias are very prevalent in children with ASD. Allik et al. [39] reported sleep patterns in school-age in children with Asperger syndrome or highfunctioning autism.

\section{ASD and Hyperexcitability and Attention Deficit (ADHD)}

We have found 10\% ASD patients with hyperactivity and attention deficit, Over the years, several authors have reported symptoms of attention deficit hyperactivity disorder (ADHD) in patients with autism spectrum disorders (ASD) [40,41]. The high presence of attention deficit hyperactivity disorder (ADHD) in autism spectrum disorder (ASD) has been acknowledged in the Diagnostic and statistical manual of mental disorders, fifth edition, thus allowing the diagnosis of both disorders [42]. According to Muskens et al. [43], the associations between neurodevelopmental disorders and personality are at least partly due to genetic 
effects influencing both conditions. We have above described the increasing relationship between ASD and ADHD mainly due to the long and systematic time using computers, tables, cellular phones and electronic plays by children giving source to an epidemic outburst of autistic children.

The high stimulation of retinal photoreceptors induces the stimulation of occipital brain cortex producing seizures, brain and language digitalization. Bektaş et al. [44] reported a prospective case-control study of autism spectrum disorder and attention-deficit/hyperactivity disorder-related symptoms in benign childhood epilepsy with centrotemporal spikes (BECTS). According to these Authors, children with typical BECTS may have an increased risk of suffering from symptoms of ASD and ADHD. Children with late onset of seizures may be more likely to develop neuropsychological disturbances regarding ASD and ADHD.

\section{ASD and Mood Disorders}

In the present study we have reported mood disorders in 3\% of ASD patients examined. We have found autistic patients with anxiety, separation anxiety, fobias, impulsivity, and desafiant negativism disorder. According to Postorino et al. [45], ASD children are at particular risk for affect and anxiety-specific problems. While symptom levels in females increase at a faster rate throughout adolescence, males with ASD appear to have elevated levels of depressive symptoms in school age that are maintained into young adulthood [46]. Autism spectrum disorder (ASD) is often associated with poor emotional control and psychopathology, such as anxiety and depression; however, little is known about the underlying mechanisms $[47,48]$. According to Morie et al. [49], individuals with autism spectrum disorder (ASD) often have co-morbid anxiety and depression. Alexithymia and emotion regulation difficulties are commonly seen in individuals with ASD and mood disorders. Alexithymia is a personality trait frequently found in adults with autism spectrum disorder (ASD), and has been linked to impairments in emotion recognition and empathy [50]. The emotion-processing profile observed in ASD might be best understood as resulting from heterogeneous vulnerabilities in different components of an "emotional communication system" that, in typical development, emerges from the interplay between domain-general cognitive, social and affective processes [51]. The emotional disorders observed in ASDs might be explained by greater skills in the processing of local information. This might explain the inability of children with ASDs to achieve coherent perception of their social environment and might also lead to the withdrawal that is characteristic of this disorder [52]. Depression and empathy were the most important predictors of disability [53]. Rates of concomitant psychiatric disorders were high and the presence of two or more co-occurring disorders was common in children with autism spectrum disorder [54]. Children with both ASD and ADHD have an increased risk of anxiety and mood disorders [55].

At the present time there is a substantial uncertainty regarding the extent and nature of autism spectrum disorder (ASD) and bipolar affective disorder (BPAD) co-occurrence, due to disparate findings in previous studies [56,57].

\section{ASD and Epilepsy}

We have found partial or focal convulsive syndromes and febrile seizures in 3\% of ASD patients. Canitano and Zappella [58] ealier described two cases of autistic epileptiform regression selected from a larger group of children with autistic spectrum disorders. Autistic regression is a well known condition that occurs in one third of children with pervasive developmental disorders, who, after normal development in the first year of life, undergo a global regression during the second year that encompasses language, social skills and play. Hartley-McAndrew and Weinstock A. [59] did not reveal statistically significant differences in behaviors between ASD children with and without EEG abnormalities. However, ASD children with seizures revealed significantly worse behaviors as compared to counterparts without seizures. According to Capal et al. [60], the association between autism spectrum disorder (ASD) and epilepsy is well-known.

Abnormalities on electroencephalography (EEG) results have been reported in patients with ASD without a history of seizures. However, little is known about the relationship between abnormalities on EEG results and the core features of ASD. El Achkar and Spence [61]. consider that the established risk factors for an increased risk of epilepsy in patients with ASD include intellectual disability and female gender. There is some evidence of an increased risk of epilepsy associated with other factors such as ASD etiology (syndromic), severity of autistic features, developmental regression, and family history. The presence of an abnormal EEG result or epilepsy in the setting of ASD suggests worse developmental and adaptive functioning. On the contrary, Milovanovic et al. [62] 2019 conclude that epilepsy, as well as subclinical epileptic discharges, showed small effects on motor skills in patients with autism, and had no effect on adaptive behavior, and communication/socialization/daily living skills. Tuchman [63] analyses the relationship of autism spectrum disorder and epilepsy and considers that the association of epilepsy and autism spectrum disorders (ASD) is best understood by examining the relationship between social cognition, nonsocial cognition, and epilepsy. The relationship between ASD and epilepsy is bidirectional and is strongly linked to intellectual disability (ID). The risk of developing ASD in children with epilepsy is highest in children with early onset seizures, with a high prevalence in children with infantile spasms. The risk of developing epilepsy in children first diagnosed with ASD is highest in those with ID. The prevalence of seizures in ASD increases with age. When epilepsy and ASD coexist, they share common pathophysiological mechanisms A high prevalence of epilepsy and interictal epileptiform discharges (IED) have been recently reported among children with ASD [62,64]. Loussouarn et al. [65] emphasized the high prevalence of EEG abnormalities in children with ASD without epilepsy, and that EEG should only be performed at epilepsy onset, and more precisely when a clinical 
interview has confirmed that repetitive paroxysmal events could be seizures. Pacheva et al. [66] postulate that in the occurrence of epilepsy among children with ASD we should define the type of epileptic seizures and syndromes, the age of onset of epilepsy, EEG abnormalities, the used antiepileptic drugs and the therapeutic responses for seizures and autistic behavior, as well as to find some correlations between epilepsy and gender, etiology and intellectual disability (ID).

\section{The Sex-Specific Pathophysiology of ASD: Autism in Males and Females}

In our study we have reported exhibiting ASD disconnected child prevalent in males than females, but the underlying neurobiology of this sex bias remains unclear. According to Smith et al. [67], given its involvement in ASD, its role in sensorimotor, cognitive, and socio-affective processes, and its developmental sensitivity to sex hormones, and the cerebellum are candidates for understanding this sex difference. These Authors used restingstate functional magnetic resonance imaging (fMRI) to investigate sex-dependent differences in cortico-cerebellar organization in ASD. Post hoc tests revealed a pattern of cortico-cerebellar hyperconnectivity in ASD females and a pattern of hypoconnectivity in ASD males. Furthermore, cortico-cerebellar FC in females more closely resembled that of control males than that of control females. These results shed light on the sex-specific pathophysiology of ASD and are indicative of potentially divergent neurodevelopmental trajectories for each sex.

\section{The Brain-Gut Relationship in ASD}

In the present paper we have reported 3\% gastrointestinal diseases in the patients examined, such as esophageal atresia and gastric reflux, constipation or diarrhea, and neonatal jaundice. New research points to a possible link between autism spectrum disorder (ASD) and the gut microbiota as many autistic children have co-occurring gastrointestinal problems. The excellent review of Srikantha and Mohajeri [68] focuses on specific alterations of gut microbiota mostly observed in autistic patients. Some other cooccurring symptoms such as mitochondrial dysfunction, oxidative stress in cells, altered tight junctions in the blood-brain barrier and structural changes in the cortex, hippocampus, amygdala and cerebellum were also detected by Srikantha and Mohajeri (2019).

In relationship among gastrointestinal symptoms, problem behaviors, and internalizing symptoms in children and adolescents with autism spectrum disorder, the majority of patients experienced constipation, about half experienced stomachaches or stomach pain, and others experienced nausea or diarrhea. Young children with aggressive problem behaviors were $11.2 \%$ more likely to have co-occurring nausea; whereas, older children showed more complex relationships between internalizing symptoms and GI symptoms [69].

\section{Autism and Parenting Social Stress}

We have above described the maternal stress factors such as labor and partnership stress. In a developing country the poverty, low economic incomes, and instability of sociopolitical situation impose additional stress inducing maternal neurobehavioral disorders. The neuropsychiatric symptoms and parenting stress are significantly higher in mothers of children with ASD, and more social supports are needed for mothers of children with ASD especially for mothers of children with low-functioning ASD [70]. Pattini et al. [71] have also related acute stress in mothers of children with autism spectrum disorder.

Social-communication skills emerge within the context of rich social interactions, facilitated by an infant's capacity to attend to people and objects in the environment. Disruption in this early neurobehavioral process may decrease the frequency and quality of social interactions and learning opportunities, potentially leading to downstream deleterious effects on social development. Bradshaw et al. [72] results provide evidence for early vulnerabilities in visual attention for infants at risk for ASD during a period of important neurodevelopmental transition (between 2 and 3 months) when attention has significant implications for social communication and cognitive development.

\section{Autism and Intelligent Fathers}

Some parents of the patients examined in the present study are highly educated and intelligent. Original case descriptions of autism noted that parents of the affected children tended to be highly educated and intelligent. Recent genetic studies indicate that risk for autism spectrum disorders (ASD) is associated with high intelligence. The relationship between paternal IQ and offspring risk of ASD was non-monotonic and varied by the presence of co-occurring disorders, probably reflecting phenotypic diversity among affected individuals [73].

\section{Maternal Infections and Diseases During Pregnancy}

We have above described the numerous pathological conditions of mothers during pregnancy considered as risks factor such as urinary infections, behavioral disturbances like anxiety and fobias, hyperactivity and attention deficit, toxoplasma and Zika virus infections, hyperemesis, oligohydramnios and loss of amniotic fluid, twin pregnancy pre-eclampsia, aging placenta, cesarean surgery, high blood pressure, maternal sepsis, diabetes and hepatic coma, hipotiroidism, viral hepatitis, obesity, and social problems, such as excessive work, low economy and poor conditions environmental contamination, labor and conjugal stress. According to Ornoy et al. [74], among the maternal diseases in pregnancy associated with ASD are diabetes mellitus (PGDM and/or GDM), some maternal autoimmune diseases like antiphospholipid syndrome (APLS) with anti-B2GP1 IgG antibodies and thyroid disease with antithyroid peroxidase (TPO) antibodies, preeclampsia and some other 
autoimmune diseases with IgG antibodies that might affect fetal brain development. Other related factors are maternal infections (rubella and CMV with fetal brain injuries, and possibly Influenza with fever, prolonged fever and maternal inflammation, especially with changes in a variety of inflammatory cytokines and antibodies that cross the placenta and affect the fetal brain.

Zerbo et al. [75]. Emphasized the maternal infection diseases. Langrigde et al. [76] included hypertension, asthma, urinary tract infection, some types of ante-partum haemorrhage, any type of preterm birth, elective C-sections, breech presentation, poor fetal growth and need for resuscitation at birth, with all factors showing an increased risk. These findings support most of pathological entities on maternal pregnancy described in the present study. In the present study we found some mother with Zika virus infection during pregnancy. Vianna et al. [77]. suggest a mechanism encompassing neurological and immunological pathways that could play a role in the development of ASD in infants after ZIKV infection in pregnancy. Recent advances in in vitro research show that ZIKV can infect and obliterate cells from the CNS, such as progenitors, neurons, and glial cells. Neural progenitor cells seem to be the main target of the virus, with infection leading to less cell migration, neurogenesis impairment, cell death and, consequently, microcephaly in newborns [78].

In a previous paper we have emphasized that the Zika virus infection during the first trimester of pregnancy evinces a greater risk of severe CNS involvement, being in the first trimester more severe damage infection than in the third trimester. Nuclear magnetic resonance neuroimages showed ventriculomegaly, absence of corpus callosum, scarce differentiation of gray and white matter, and vascular and cerebellar hypoplasia [79]. We herein reporting the toxoplasma gondi infection in mothers with ASD child. We have not found thus far previous reports dealing with this apparently risk factor. In the present study some mothers receive cesarean surgery exposing the children to the effects of anesthetic. Further research studies are needed to establish the relationship among neural connectivity, cesarean surgery, autism and anesthetics.

\section{Immune Dysfunction in ASD}

We have herein reported allergic diseases, such as bronchial asthma and atopic dermatitis (3\%) in ASD patients. Dysregulation in immune responses during pregnancy increases the risk of a having a child with an autism spectrum disorder (ASD). Asthma is one of the most common chronic diseases among pregnant women, and symptoms often worsen during pregnancy [80]. Two main immune dysfunctions in autism are immune regulation involving pro-inflammatory cytokines and autoimmunity. Studies showing elevated brain specific antibodies in autism support an autoimmune mechanism. Viruses may initiate the process, but the subsequent activation of cytokines is the damaging factor associated with autism. Virus specific antibodies associated with measles virus have been demonstrated in autistic subjects. Maternal antibodies may trigger autism as a mechanism of autoimmunity. MMR vaccination may increase risk for autism via an autoimmune mechanism in autism [81]. Epidemiological studies have shown a relationship with maternal immune disturbances during pregnancy and ASD. Moreover, decades of research have identified numerous systemic and cellular immune abnormalities in individuals with ASD and their families. These include changes in immune cell number, differences in cytokine and chemokine production, and alterations of cellular function at rest and in response to immunological challenge. Many of these changes in immune responses are associated with increasing impairment in behaviors that are core features of ASD [82-84].

Individuals diagnosed with ASD have alterations in immune cells such as T cells, B cells, monocytes, natural killer cells, and dendritic cells. Also, many individuals diagnosed with ASD have alterations in immunoglobulins and increased autoantibodies. Finally, an important portion of individuals diagnosed with ASD has elevated peripheral cytokines and chemokines and associated neuroinflammation [85]. Scientific research studies emerging within the past two decades suggest that immune dysfunction and inflammation have pathogenic influences through different mechanisms, all leading to both a chronic state of low grade inflammation, and alterations in the central nervous system and immune response, respectively [86]. Neuro-inflammation and neuro-immune abnormalities have now been established in ASD as key factors in its development and maintenance [87].

Inflammation in the brain and CNS has been reported by several groups with notable microglia activation and increased cytokine production in postmortem brain specimens of young and old individuals with ASD [88]. Further, it is the heterogeneity within this disorder that has brought to light much of the research emerging within the past two decades suggests that immune dysfunction is a viable risk factor contributing to the neurodevelopmental deficits observed in autism spectrum disorders (ASD). During gestation, prenatal insults including maternal infection and subsequent immunological activation may increase the risk of autism in the child. Similarly, the presence of maternally derived anti-brain autoantibodies found in $\sim 20 \%$ of mothers whose children are at risk for developing autism has defined an additional subphenotype of ASD [89].

According to Ahmad et al. [90], dysregulation of $\mathrm{T}$ cell immunoglobulin and mucin domain 3 (TIM-3) signaling in peripheral immune cells is associated with immune dysfunction in autistic children. Mothers of children with autism spectrum disorder (ASD) are significantly more likely to have an autoimmune disease than women of neurotypically developing children. According to Gata-Garcia and Diamond [91], these evidences support a contribution of maternal brain-reactive antibodies to neurodevelopmental disorders. An interplay between antibodies, genetics, and other environmental factors is likely to determine the specific neurodevelopmental phenotypes and their severity. 
Additional modulating factors likely also include the microbiome, sex chromosomes, and gonadal hormones. These interactions may help to explain the sex-bias observed in neurodevelopmental disorders and support a contribution of maternal brain-reactive antibodies to neurodevelopmental disorders. According with AbouDonia et al. [92] (2019) study of autoantibodies against neuronal and glial proteins in ASD children and mothers, the levels of circulating IgG class autoantibodies against the nine proteins were significantly elevated in ASD children. Mothers of ASD children exhibited increased levels of autoantibodies against all panel of tested proteins except for S100B and tubulin compared to agematched healthy control children and their mothers.

Control children and their mothers showed low and insignificant levels of autoantibodies to neuronal and glial proteins. These results strongly support the importance of anti-neuronal and glial protein autoantibodies biomarker in screening for ASD children and further confirm the importance of the involvement of the maternal immune system as an index that should be considered in fetal in utero environmental exposures. Mast cell activation by allergic, infectious, environmental and stress-related triggers, especially perinatally, would release pro-inflammatory and neurotoxic molecules. We speculate these could disrupt the gut-blood-brain barriers, thus contributing to brain inflammation and ASD pathogenesis. Increased mast cell responsiveness may define at least a subgroup of ASD subjects, who could benefit from inhibition of mast cell activation [93].

Autoantibodies against brain epitopes in mothers of children with ASD and many such children strongly correlate with allergic symptoms and indicate an aberrant immune response, as well as disruption of the blood-brain barrier (BBB). Recent epidemiological studies have shown a strong statistical correlation between risk for ASD and either maternal or infantile atopic diseases, such as asthma, eczema, food allergies and food intolerance, all of which involve activation of mast cells (MCs). These unique tissue immune cells are located perivascularly in all tissues, including the thalamus and hypothalamus, which regulate emotions. MC-derived inflammatory and vasoactive mediators increase BBB permeability [94-96]. Mast cells (MCs) are located perivascularly close to neurons and microglia, primarily in the leptomeninges, thalamus, hypothalamus and especially the median eminence.

Corticotropin-releasing factor (CRF) is secreted from the hypothalamus under stress and, together with neurotensin (NT), can stimulate brain MCs to release inflammatory and neurotoxic mediators that disrupt the blood-brain barrier (BBB), stimulate microglia and cause focal inflammation. CRF and NT synergistically stimulate MCs and increase vascular permeability; these peptides can also induce each other's surface receptors on MCs leading to autocrine and paracrine effects. As a result, brain MCs may be involved in the pathogenesis of "brain fog," headaches, and autism spectrum disorders (ASDs), which worsen with stress. CRF and NT are significantly increased in serum of ASD children compared to normotypic controls further strengthening their role in the pathogenesis of autism [97].

\section{The Genetic Architecture of ASD}

The genetic architecture of ASD has become increasingly clear and increasingly complex with estimates of at least 1000 genetic alterations associated with the risk for ASD [4]. In the last 10 years, there have been significant advances in understanding the genetic basis for ASD, critically supported through the establishment of ASD bio-collections and application in research. Collectively, these include mapping ASD candidate genes, assessing the nature and frequency of gene mutations and their association with ASD clinical subgroups, insights into related molecular pathways such as the synapses, chromatin remodelling, transcription and ASD-related brain regions [98]. Multiple lines of evidence from genetic linkage studies to animal models implicate aberrant cortical plasticity and metaplasticity in the pathophysiology of autism spectrum disorder (ASD) and fragile X syndrome (FXS) [99].

Data from whole-genome screens in multiplex families suggest interactions of at least 10 genes in the causation of autism. Thus far, a putative speech and language region at 7q31-q33 seems most strongly linked to autism, with linkages to multiple other loci under investigation. Cytogenetic abnormalities at the 15q11-q13 locus are fairly frequent in people with autism, and a "chromosome 15 phenotype" was described in individuals with chromosome 15 duplications. Among other candidate genes are the FOXP2, RAY1/ ST7, IMMP2L, and RELN genes at 7q22-q33 and the GABA(A) receptor subunit and UBE3A genes on chromosome 15q11-q13 [100].

Prior structural MRI studies demonstrated atypical gray matter characteristics in siblings of individuals with autism spectrum disorder (ASD). However, they did not clarify which aspect of gray matter is related to the endophenotype (i.e. genetic vulnerability) of ASD. This proof-of-concept study suggests that an ASD endophenotype emerges in sulcal depth SD and that neural bases for ASD diagnosis can be discerned from the endophenotype when accounted for the difference between TD siblings [101].

Lin et al. [102] sub-categorize risk genes that affect the structural connectivity of neurons into

a) Cytoskeletal regulators, e.g., motors and small RhoGTPase regulators;

b) Adhesion molecules, Besides, NCAM, and neurexin superfamily;

c) Cell surface receptors, e.g., glutamatergic receptors and receptor tyrosine kinases;

d) Signalingmolecules, e.g.,protein kinases and phosphatases and

e) Synaptic proteins, e.g., vesicle and scaffolding proteins. 
These Authors reported high comorbidity, sequential risk, and shared familial risks between obsessive-compulsive disorders (OCD) and autism spectrum disorders, which are suggestive of partially shared etiological mechanisms. The results have implications for current gene-searching efforts and for clinical practice.

\section{Mitochondrial Dysfunction in Children with ASD}

The different clinical symptoms found in ASD patients as observed in the presentstudy suggest the dysfunction of a cell energy organelle as mitochondria. Rose et al. [6]. Systematically review the literature on human studies of mitochondrial dysfunction related to ASD. Clinical aspects of mitochondrial dysfunction in ASD include unusual neurodevelopmental regression, especially if triggered by an inflammatory event, gastrointestinal symptoms, seizures, motor delays, fatigue and lethargy. Recent researches have revealed the influence of mitochondrial physiology on the development of ASD. Several research groups have identified defects in respiratory complexes, coenzyme-Q10 deficiency, increased oxidative damage, decreased of superoxide dismutase (SOD2). A study on the influence of mitochondrial physiology on the development of ASD can provide new alternatives and challenges. The increment of mitochondrial DNA, high oxidative stress, and high expression of the MFN2 gene could help as a scanner of the mitochondrial function in children with ASD [103].

\section{Neuroimaging-Based Neuroanatomical Studies of ASD}

Significant heterogeneity across aetiologies, neurobiology and clinical phenotypes have been observed in individuals with autism spectrum disorder (ASD). Neuroimaging-based neuroanatomical studies of ASD have often reported inconsistent findings which may, in part, be attributable to an insufficient understanding of the relationship between factors influencing clinical heterogeneity and their relationship to brain anatomy. Magnetic resonance images have shown greater cortical thickness in sex specific regions; further, cortical thickness differences were observed to be greater in younger individuals and in those with sex, age and full-scale intelligence (FIQ) lower and to be related to neuroanatomical heterogeneity and overall clinical severity. [104].

\section{Neuropathological Findings and Brain Overgrowth in ASD}

Neuropathological studies appear to have identified common abnormalities in the cerebellum and limbic system, marked Purkinje cell loss in the cerebellar hemispheres, together with retained fetal neuronal circuitry in cerebellar nuclei and increased neuronal packing in specific regions of the limbic system, amygdala, and hippocampus [105] The most replicated neuroanatomical finding in autism is the tendency toward brain overgrowth, especially in younger children. Research shows that both gray and white matter are enlarged. Proposed mechanisms underlying brain enlargement include abnormal inflammatory and neurotrophic signals that lead to excessive, aberrant dendritic connectivity via disrupted pruning and cell adhesion, and enlargement of white matter due to excessive gliogenesis and increased myelination. Amyloid- $\beta$ protein precursor ( $\beta$ APP) and its metabolites, more commonly associated with Alzheimer's disease (AD), are also dysregulated in autism plasma and brain tissue samples [106].

\section{Synaptopathology Involved in Autism Spectrum Disorder}

Multiple studies have revealed that mutations ingenes like NRXN, NLGN, SHANK, TSC1/2, FMR1, and MECP2 converge on common cellular pathways that intersect at synapses. These genes encode cell adhesion molecules, scaffolding proteins and proteins involved in synaptic transcription, protein synthesis and degradation, affecting various aspects of synapses including synapse formation and elimination, synaptic transmission and plasticity. This suggests that the pathogenesis of ASD may, at least in part, be attributed to synaptic dysfunction [107]. Most ASD genes are implicated in neurogenesis, structural maturation, synaptogenesis and function [108] SHANK3 is a synaptic scaffolding protein localized in the postsynaptic density and has a crucial role in synaptogenesis and neural physiology. Deletions and point mutations in SHANK3 cause Phelan-McDermid Syndrome (PMS) and have also been implicated in autism spectrum disorder (ASD) and intellectual disabilities, leading to the hypothesis that reduced SHANK3 expression impairs basic brain functions that are important for social communication and cognition.

\section{Some Neurochemical Research in ASD}

Pavăl [109] has proposed a dopamine hypothesis of autism spectrum disorder. that autistic behavior arises from dysfunctions in the midbrain dopaminergic system and that a dysfunction of the mesocorticolimbic circuitleads to social deficits, while a dysfunction of the nigrostriatal circuit leads to stereotyped behaviors. This hypothesis is based clinical studies of dopamine antagonists which seem to have improving effects on autistic behavior. The 5-HT transporter (SERT) gene (SLC6A4) has been associated with whole blood 5-HT levels and ASD susceptibility. strong support for the hypothesis that altered 5-HT homeostasis can impact risk for ASD traits and provide a model with construct and face validity that can support further analysis of ASD mechanisms and potentially novel treatments [110].

Elevated whole blood serotonin, or hyperserotonemia, was the first biomarker identified in autism spectrum disorder (ASD) and is present in more than $25 \%$ of affected children. The serotonin system is a logical candidate for involvement in ASD due to its pleiotropic role across multiple brain systems both dynamically and across development Emerging data from both neuroimaging and postmortem samples also indicate changes in the brain serotonin system in ASD. Genetic linkage and association studies of both whole blood serotonin levels and of ASD risk point to the chromosomal region containing the serotonin transporter (SERT) gene in males but not in females. In ASD families with evidence of linkage to this region, multiple rare SERT amino acid variants lead to a convergent increase in serotonin uptake in cell models [111]. 
Children with ASD tend to have higher free radicals than antioxidants compared with their matched controls. This disequilibrium of oxidative stress has been associated with the pathogenesis of this neurocognitive disorder [112]. Gilbert et al. [113] formulate the hypothesis that there may be a link between cholesterol, vitamin D, and steroid hormones which subsequently impacts on the development of at least some of patients with ASD. Our hypothesis, posits that there may be links between cholesterol metabolism, which we will refer to as "steroid metabolism" and findings of steroid abnormalities of various kinds (cortisol, testosterone, estrogens, progesterone, vitamin D) in autism spectrum disorder (ASD). Urea cycle disorder was also identified, namely, ornithine transcarbamylase deficiency and arginase deficiency, because of the high level of ammonia in the patient's bloodstream [114].

\section{Conclusion}

We have examined 75 infant patients from 3 to 15 years old exhibiting disconnected brain with social isolation $(80 \%)$, hyperconnected children (5\%), creative child, (Asperger syndrome) (10\%), stereotypic movements of hands and body (10\%), psicomotor retard (5\%), behavioral changes such as aggresivity and autoagressivity $(10 \%)$, crisis of tears $(1 \%)$, separation anxiety $(1 \%)$, mood disorders ( $3 \%)$, photofobia (1\%), loss of weight, (1\%), routinised patterns of though and fantastic thoughts (2\%), language disorders such as delay in the onset of language or early vocalization, regressive changes of language, mutism, gestual language, escatologic language, digital language (5\%), learning and memory deficit (10\%). The following associated comorbidities: perinatal hypoxia, low weight at birth, behavioral abnormalities, anxiety, auto- and heteroaggressivity, language disorders, hiperphagia, learning and memory deficit, hearing disorders, mainly hiperacusia, Social isolation, cognitive deficit, sleeping disorders and parenteral abuse of child. Some non-nervous system comorbidities, such as pulmonary diseases and allergic reactions also were found. Some locomotor abnormalities as genus valgo and flat feet were also observed. The mothers exhibited the followings diseases during pregnancy: urinary infections, behavioral disturbances like anxiety, fobias, hyperactivity, toxoplasmosis and Zika virus infections, hyperemesis, oligohydramnios and loss of amniotic fluid, twin pregnancy, pre-eclampsia, aging placenta, cesarean, high blood pressure, maternal sepsis, diabetes, hepatic coma, hipotiroidism, viral hepatitis, parent obesity, and social problems, such as excessive work, low economy and poor social conditions, environmental contamination and labor and conjugal stress. We emphasized different phenotype subtypes of ASD related with the environmental changes of developing countries and the multiple maternal pathology as risks factor for ASD.

\section{Acknowledgement}

The present study has been carried out by a subvention obtained from Castejón Foundation and the logistic support of Biological Research Institute. Faculty of Medicine. Zulia University and Clinical Neuroscience Institute. San Rafael Home Clinic Maracaibo. Venezuela.

\section{References}

1. Gottfried C, Bambini Junior V, Francis F, Riesgo R, Savino W (2017) The impact of neuroimmune alterations in autism spectrum disorder. Brain Connect 7(9): 558-573.

2. Bölte S, Girdler S, Marschik PB (2019) The contribution of environmental exposure to the etiology of autism spectrum disorder. Cell Mol Life Sci 76(7): 1275-1297.

3. Ng M, Montigny JG, Ofner M, Docé MT (2017) Environmental factors associated with autism spectrum disorder: a scoping review for the years 2003-2013. Health Promot Chronic Dis Prev Can 37(1): 1-23.

4. Beversdorf DQ Missouri Autism Summit Consortium (2016) Phenotyping, etiological factors, and biomarkers: toward precision medicine in autism spectrum disorders. J Dev Behav Pediatr 37(8): 659673.

5. Von Ehrenstein OS, Ling C, Cui,X, Cockburn M, Park AS, et al. (2019) Prenatal and infant exposure to ambient pesticides and autism spectrum disorder in children: population based case-control study. BMJ 364: 1962.

6. Rose S, Niyazov DM, Rossignol DA, Goldenthal M, Kahler SG, et al. (2018) Clinical and molecular characteristics of mitochondrial dysfunction in autism spectrum disorder. Mol Diagn Ther 22(5): 571-593.

7. Tye C, Abigail K Runicles, Whitehouse AJO, Alvares GA (2018) Characterizing the interplay between autism spectrum disorder and comorbid medical conditions: An integrative review Front Psychiatry 9: 751.

8. Cerliani, L Mennes M, Thomas RM, Di Martino A, Thioux M, et al. (2015) Increased functional connectivity between subcortical and cortical resting-state networks in autism spectrum disorder. JAMA Psychiatry 72(8): 767-777.

9. Gotham K, Brunwasser SM, Lord C (2015) Depressive and anxiety symptom trajectories from school age through young adulthood in samples with autism spectrum disorder and developmental delay. J Am Acad Child Adolesc Psychiatry 54(5): 369-376.

10. Castejon OJ, Galindez P, Torres IA, Leal J, Villasmil A, et al. (2018) Maternal Stress, Pregnancy Diseases and Child Hyperactivity and Attention Deficit (ADHD). Biomedical Journal of Scientific \& Technical Research.

11. Wilkes BJ, Lewis MH (2018) The neural circuitry of restricted repetitive behavior: Magnetic resonance imaging in neurodevelopmental disorders and animal models. Neurosci Biobehav Rev 92: 152-171.

12. Cai J, Hu X, Guo K, Yang P, Situ M, et al. (2018) Increased left inferior temporal gyrus was found in both low function autism and high function autism. Front Psychiatry 9: 542

13. Puts NAJ, Wodka EL, Harris AD, Crocetti D, Tommerdahl M, et al. (2017) Reduced GABA and altered somatosensory function in children with autism spectrum disorder. Autism Res 10(4): 608-619.

14. Sapey Triomphe LA, Lamberton F, Sonié S, Mattout J, Schmitz C (2019) Tactile hypersensitivity and GABA concentration in the sensorimotor cortex of adults with autism. Autism Res 12(4): 562-575.

15. Bjørklund G (1998) Children with Asperger syndrome. Tidsskr Nor Laegeforen 118(10): 1567-1569.

16. Huang MX, Liu XH, Zhang ZJ, Chen C, Wang D, et al. (2018) Functional connection between the stereotyped behavior and the motor front area in children with autism. Br J Neurosurg 11: 1-4.

17. Abbott AE, Nair A, Keown CL, Datko M, Jahedi A, et al. (2016) Patterns of atypical functional connectivity and behavioral links in autism differ between default, salience, and executive networks. Cereb Cortex 26(10): 4034-4045.

18. Rinehart NJ, Bradshaw JL, Brereton AV, Tonge BJ (2002) A clinical and neurobehavioural review of high-functioning autism and Asperger's disorder. Aust N Z J Psychiatry 36(6): 762-770. 
19. Macintosh KE, Dissanayake C (2004) The similarities and differences between autistic disorder and Asperger's disorder: a review of the empirical evidence. J Child Psychol Psychiatry 45(3): 421-434.

20. Takahata K , Kato M (2008) Neural mechanism underlying autistic savant and acquired savant syndrome. Brain Nerve 60(7): 861-869.

21. Paquet A, Olliac B, Bouvard MP, Golse B, Vaivre Douret L (2016) The Semiology of motor disorders in autism spectrum disorders as highlighted from a standardized neuro-psychomotor assessment. Front Psychol 12(7): 1292.

22. Paquet A, Olliac B, Golse B, Vaivre Douret L (2019) Nature of motor impairments in autism spectrum disorder: A comparison with developmental coordination disorder. J Clin Exp Neuropsychol 41(1): $1-14$

23. Shevell MI, Majnemer A, Rosenbaum P, Abrahamowicz M (2000) Etiologic yield of subspecialists' evaluation of young children with global developmental delay. J Pediatr 136(5): 593-598.

24. Foss Feig JH, Schauder KB, Key AP, Wallace MT, Stone WL (2017) Audition-specific temporal processing deficits associated with language function in children with autism spectrum disorder. Autism Res 10(11) 1845-1856.

25. Sharda M, Foster NEV, Tryfon A, Doyle Thomas KAR, Ouimet T, et al. (2017) Language ability predicts cortical structure and covariance in boys with autism spectrum disorder. Cereb Cortex 27(3): 1849-1862.

26. Lo YC, Chou TL, Fan LY, Gau SS, Chiu YN, et al. (2013) Altered structurefunction relations of semantic processing in youths with high-functioning autism: a combined diffusion and functional MRI study. Autism Res 6(6): 561-570.

27. Baum SH, Stevenson RA, Wallace MT (2015) Behavioral, perceptual, and neural alterations in sensory and multisensory function in autism spectrum disorder. Prog Neurobiol 134: 140-160.

28. Riquelme I, Hatem SM, Montoya P (2016) Abnormal pressure pain, touch sensitivity, proprioception, and manual dexterity in children with autism spectrum disorders. Neural Plast.

29. Baranek GT, Woynaroski TG, Nowell S, Turner Brown, L, DuBay M, et al. (2018) Cascading effects of attention disengagement and sensory seeking on social symptoms in a community sample of infants at-risk for a future diagnosis of autism spectrum disorder. Dev Cogn Neurosci 29: 30-40.

30. Mikkelsen M, Wodka EL, Mostofsky SH, Puts NAJ (2018) Autism spectrum disorder in the scope of tactile processing. Dev Cogn Neurosci 29: $140-150$

31. Copping NA, Berg EL, Foley GM, Schaffler MD, Onaga BL, et al. (2017) Touchscreen learning deficits and normal social approach behavior in the Shank3B model of Phelan-McDermid Syndrome and autism. Neuroscience 345: 155-165.

32. Erdődi L, Lajiness O Neill R, Schmitt TA (2013) Learning curve analyses in neurodevelopmental disorders: are children with autism spectrum disorder truly visual learners? J Autism Dev Disord 43(4): 880-890.

33. Koller HP (2012) Visual processing and learning disorders. Curr Opin Ophthalmol 23(5): 377-383.

34. Kantzer AK, Fernell E, Gillberg C, Miniscalco C (2013) Autism in community pre-schoolers: developmental profiles. Res Dev Disabil 34(9): 2900-2908.

35. Burrows EL, Laskaris L, Koyama L, Churilov L, Bornstein JC, et al. (2015) A neuroligin-3 mutation implicated in autism causes abnorma aggression and increases repetitive behavior in mice. Mol Autism 6: 62.

36. Yamamuro K, Tsujii N, Ota T, Kishimoto T, Iida J (2017) Pharmacotherapy for the treatment of aggression in pediatric and adolescent patients with autism spectrum disorder comorbid with attention-deficit hyperactivity disorder: A questionnaire survey of 571 psychiatrists. Psychiatry Clin Neurosci 71(8): 554-561.

37. Sener EF, Taheri S, Sahin MC, Bayramov KK, Marasli MK, et al. (2019) Altered global mRNA expressions of pain and aggression related genes in the blood of children with autism spectrum disorders. J Mol Neurosci 67(1): 89-96.

38. Liu X, Hubbard JA, Fabes RA, Adam JB (2006) Sleep disturbances and correlates of children with autism spectrum disorders. Child Psychiatry Hum Dev 37(2): 179-191.

39. Allik H, Larsson J0, Smedje H (2006) Sleep patterns of school-age children with Asperger syndrome or high-functioning autism. J Autism Dev Disord 36(5): 585-595.

40. Lamanna AL, Craig F, Matera E, Simone M, Buttiglione M, et al. (2017) Risk factors for the existence of attention deficit hyperactivity disorder symptoms in children with autism spectrum disorders. Neuropsychiatr Dis Treat 13: 1559-1567.

41. Mansour R, Dovi AT, Lane DM, Loveland KA, Pearson DA (2017) ADHD severity as it relates to comorbid psychiatric symptomatology in children with Autism Spectrum Disorders (ASD). Res Dev Disabil 60: 52-64.

42. Berenguer Forner C, Miranda Casas A, Pastor Cerezuela G, Roselló Miranda R (2015) Comorbidity of autism spectrum disorder and attention deficit with hyperactivity. A review study. Rev Neurol 60(1): 37-43.

43. Muskens JB, Velders FP, Staal WG (2017) Medical comorbidities in children and adolescents with autism spectrum disorders and attention deficit hyperactivity disorders: a systematic review. Eur Child Adolesc Psychiatry 26(9): 1093-1103.

44. Bektaş G, Tekin U, Yıldız EP, Aydınlı N, Calışkan M, et al. (2019) Autism spectrum disorder and attention deficit/hyperactivity disorder-related symptoms in benign childhood epilepsy with centro-temporal spikes: A prospective case-control study. Epilepsy Behav 95: 61-64.

45. Postorino V, Kerns CM, Vivanti G, Bradshaw J, Siracusano M, et al. (2017) Anxiety disorders and obsessive-compulsive disorder in individuals with autism spectrum disorder. Curr Psychiatry Rep 19(12): 92.

46. Gaigg SB (2012) The Interplay between emotion and cognition in autism spectrum disorder: Implications for Developmental Theory. Front Integr Neurosci 6: 113

47. Skokauskas N, Gallagher L (2010) Psychosis, affective disorders and anxiety in autistic spectrum disorder: prevalence and nosological considerations. Psychopathology 43(1): 8-16.

48. Mazefsky CA, Borue X, Day TN, Minshew NJ (2014) Emotion regulation patterns in adolescents with high-functioning autism spectrum disorder: comparison to typically developing adolescents and association with psychiatric symptoms. Autism Res 7(3): 344-354.

49. Morie KP, Jackson S, Zhai ZW, Potenza MN, Dritschel B (2019) Mood Disorders in High-Functioning Autism: The Importance of Alexithymia and Emotional Regulation. J Autism Dev Disord 49(7): 2935-2945.

50. Milosavljevic B, Carter Leno V, Simonoff E, Baird G, Pickles A, et al. (2016) Alexithymia in adolescents with autism spectrum disorder: its relationship to internalising difficulties, sensory modulation and social cognition. J Autism Dev Disord 46(4): 1354-1367.

51. Nuske HJ, Vivanti G, Dissanayake C (2013) Are emotion impairments unique to, universal, or specific in autism spectrum disorder? A comprehensive review. Cogn Emot 27(6): 1042-1061.

52. Meaux E, Gillet P, Bonnet Brilhault F, Barthélémy C, Batty M (2011) Atypical perception processing and facial emotion disorder in autism. Encephale 37(5): 371-378.

53. Pepper KL, Demetriou EA, Park SH, Song YC, Hickie IB, et al. (2018) Autism, early psychosis, and social anxiety disorder: understanding the role of social cognition and its relationship to disability in young adults with disorders characterized by social impairments. Transl Psychiatry 8(1): 233.

54. Lecavalier L, McCracken CE, Aman MG, McDougle CJ, McCracken JT, et al. (2019) An exploration of concomitant psychiatric disorders in children with autism spectrum disorder. Compr Psychiatry 88: 57-64.

55. Gordon Lipkin E, Marvin AR, Law JK, Lipkin PH (2018) Anxiety and mood disorder in children with autism spectrum disorder and ADHD. Pediatrics 141(4): e20171377. 
56. Skokauskas N, Gallagher L (2010) Psychosis, affective disorders and anxiety in autistic spectrum disorder: prevalence and nosological considerations. Psychopathology 43(1): 8-16.

57. Skokauskas N, Frodl T (2015) Overlap between autism spectrum disorder and bipolar affective disorder. Psychopathology 48(4): 209216.

58. Canitano R, Zappella M (2006) Autistic epileptiform regression. Funct Neurol 21(2): 97-101.

59. Hartley McAndrew M, Weinstock A (2010) Autism Spectrum Disorder: Correlation between aberrant behaviors, EEG abnormalities and seizures. Neurol Int 2(1): e10.

60. Capal JK, Carosella C, Corbin E, Horn PS, Caine R, et al. (2018) EEG endophenotypes in autism spectrum disorder. Epilepsy Behav 88: 341348.

61. El Achkar CM, Spence SJ (2015) Clinical characteristics of children and young adults with co-occurring autism spectrum disorder and epilepsy. Epilepsy Behav 47: 183-190.

62. Milovanovic M, Radivojevic V, Radosavljev Kircanski J, Grujicic R, Toskovic 0, et al. (2019) Epilepsy and interictal epileptiform activity in patients with autism spectrum disorders. Epilepsy Behav 92: 45-52.

63. Tuchman R (2017) What is the relationship between autism spectrum disorders and epilepsy? Semin Pediatr Neurol 24(4): 292-300.

64. Anukirthiga B, Mishra D, Pandey S, Juneja M, Sharma N (2019) Prevalence of epilepsy and inter-ictal epileptiform discharges in children with autism and attention-deficit hyperactivity disorder. Indian J Pediatr.

65. Loussouarn A, Dozières Puyravel B, Auvin S (2019) Autistic spectrum disorder and epilepsy: diagnostic challenges. Expert Rev Neurother 19(6): 579-585.

66. Pacheva I, Ivanov I, Yordanova R, Gaberova K, Galabova F, et al. (2019) Epilepsy in children with autistic spectrum disorder. Children (Basel) 6(2): 15.

67. Smith REW, Avery JA, Wallace GL, Kenworthy L, Gotts SJ, et al. (2019) Sex differences in resting-state functional connectivity of the cerebellum in autism spectrum disorder. Front Hum Neurosci 13: 104.

68. Srikantha P, Mohajeri MH (2019) The possible role of the microbiotagut-brain-axis in autism spectrum disorder. Int J Mol Sci 20(9): E2115.

69. Ferguson BJ, Dovgan K, Takahashi N, Beversdorf DQ (2019) The relationship among gastrointestinal symptoms, problem behaviors, and internalizing symptoms in children and adolescents with autism spectrum disorder. Front Psychiatry 10: 194.

70. Huang MX, Liu XH, Zhang ZJ, Chen C, Wang D, et al. (2018) Functional connection between the stereotyped behavior and the motor front area in children with autism. Br J Neurosurg 11: 1-4.

71. Pattini E, Carnevali L, Troisi A, Matrella G, Rollo D, et al. (2019) Psychological characteristics and physiological reactivity to acute stress in mothers of children with Autism Spectrum Disorder. Stress Health.

72. Bradshaw J, Klin A, Evans L, Klaiman C, Saulnier C, et al. (2019) Development of attention from birth to 5 months in infants at risk for autism spectrum disorder. Dev Psychopathol 23: 1-11.

73. Gardner RM, Dalman C, Rai MRCPsych D, Lee BK, Karlsson H (2019) The Association of paternal IQ with autism spectrum disorders and its comorbidities: A population-based cohort study. J Am Acad Child Adolesc Psychiatry.

74. Ornoy A, Weinstein Fudim L, Ergaz Z (2016) Genetic syndromes, maternal diseases and antenatal factors associated with autism spectrum disorders (ASD). Front Neurosci 10: 316.

75. Zerbo O, Qian Y, Yoshida C, Fireman BH, Klein NP, et al. (2017) Association between influenza infection and vaccination during pregnancy and risk of autism spectrum disorder. JAMA Pediatr 171(1): e163609.

76. Langridge AT, Glasson EJ, Nassar N, Jacoby P, Pennell C, et al. (2013) Maternal conditions and perinatal characteristics associated with autism spectrum disorder and intellectual disability. PLoS One 8(1): e50963.

77. Vianna P, Gomes JDA, Boquett JA, Fraga LR, Schuch JB, et al. (2018) Zika Virus as a possible risk factor for autism spectrum disorder: Neuroimmunological Aspects. Neuroimmunomodulation 25(5-6): 320327.

78. Russo FB, Jungmann P, Beltrão Braga PCB (2017) Zika infection and the development of neurological defects. Cell Microbiol 19(6).

79. Castejon OJ, Torres IA, Finol P, Galindez P, Castejon Salones M, et al. (2019) Congenital Zika Sindrome. A clinical study of six patients. Open Acces J Neurol Neurosurg Juniper Publisher, USA.

80. Vogel Ciernia A, Careaga M, LaSalle JM, Ashwood P (2018) Microglia from offspring of dams with allergic asthma exhibit epigenomic alterations in genes dysregulated in autism. Glia 66(3): 505-521.

81. Cohly HH, Panja A (2005) Immunological findings in autism. Int Rev Neurobiol 71: 317-341.

82. Careaga M, Ashwood P (2012) Autism spectrum disorders: from immunity to behavior. Methods Mol Biol 934: 219-240.

83. Hsiao EY (2013) Immune dysregulation in autism spectrum disorder. Int Rev Neurobiol 113: 269-302.

84. Noriega DB, Savelkoul HF (2014) Immune dysregulation in autism spectrum disorder. Eur J Pediatr 173(1): 33-43.

85. Dipasquale V, Cutrupi MC, Colavita L, Manti S, Cuppari C, et al. (2017) Neuroinflammation in Autism Spectrum Disorders: Role of High Mobility Group Box 1 Protein. Int J Mol Cell Med 6(3): 148-155

86. Siniscalco D, Schultz S, Brigida AL, Antonucci N (2018) Inflammation and neuro-immune dysregulations in autism spectrum disorders. Pharmaceuticals (Basel) 11(2): E56.

87. Gesundheit B, Rosenzweig JP, Naor D, Lerer B, Zachor DA, et al. (2013) Immunological and autoimmune considerations of autism spectrum disorders. J Autoimmun 44: 1-7.

88. Meltzer A, Van de Water J (2017) The role of the immune system in autism spectrum disorder. Neuropsychopharmacology 42(1): 284-298.

89. Ahmad SF, Ansari MA, Nadeem A, Bakheet SA, Al Ayadhi LY, et al. (2019) Dysregulation of $\mathrm{T}$ cell immunoglobulin and mucin domain 3 (TIM3) signaling in peripheral immune cells is associated with immune dysfunction in autistic children. Mol Immunol 106: 77-86.

90. Gata Garcia A, Diamond B (2019) Maternal Antibody and ASD: Clinical Data and Animal Models. Front Immunol 10: 1129.

91. Abou Donia MB, Suliman HB, Siniscalco D, Antonucci N, El Kafrawy P (2019) De novo blood biomarkers in autism: autoantibodies against neuronal and glial proteins. Behav Sc (Basel) 9(5): E47.

92. Angelidou A, Alysandratos KD, Asadi S, Zhang B, Francis K, et al. (2011) Brief report: "allergic symptoms" in children with Autism Spectrum Disorders. More than meets the eye? J Autism Dev Disord 41(11): 15791585.

93. Angelidou A, Asadi S, Alysandratos KD, Karagkouni A, Kourembanas S, et al. (2012) Perinatal stress, brain inflammation and risk of autism-review and proposal. BMC Pediatr 12: 89.

94. Theoharides TC (2013) Is a subtype of autism an allergy of the brain? Clin Ther 35(5): 584-591.

95. Theoharides TC, Tsilioni I, Patel AB, Doyle R (2016) Atopic diseases and inflammation of the brain in the pathogenesis of autism spectrum disorders. Transl Psychiatry 6(6): e844.

96. Theoharides TC, Stewart JM, Panagiotidou S, Melamed I (2016) Mast cells, brain inflammation and autism. Eur J Pharmacol 778: 96-102.

97. Reilly J, Gallagher L, Chen JL, Leader G, Shen S (2017) Bio-collections in autism research. Mol Autism 8: 34.

98. Oberman LM, Fritz Ifert Miller F, Najib U, Shahid Bashir S, Gonzalez Heydrich J, et al. (2016) Abnormal mechanisms of plasticity and metaplasticity in autism spectrum disorders and fragile x syndrome. J Child Adolesc Psychopharmacol 26(7): 617-624. 
99. Muhle R, Trentacoste SV, Rapin I (2004) The genetics of autism. Pediatrics 113(5): 472-486.

100. Yamagata B, Itahashi T, Fujino J, Ohta H, Takashio O, et al. (2019) Cortical surface architecture endophenotype and correlates of clinical diagnosis of autism spectrum disorder. Psychiatry Clin Neurosci.

101. Lin YC, Frei JA, Kilander MB, Shen W, Blatt GJ (2016) A Subset of Autism-Associated Genes Regulate the Structural Stability of Neurons. Front Cell Neurosci 10: 263.

102. Carrasco M, Salazar C, Tiznado W, Ruiz LM (2019) Alterations of mitochondrial biology in the oral mucosa of chilean children with autism spectrum disorder (ASD). Cells 8(4): E367.

103. Bedford SA, Park MTM, Devenyi GA, Tullo S, Germann J, et al. (2019) Large-scale analyses of the relationship between sex, age and intelligence quotient heterogeneity and cortical morphometry in autism spectrum disorder. J Mol Psychiatry.

104. Lotspeich LJ, Ciaranello RD (1993) The neurobiology and genetics of infantile autism. Int Rev Neurobiol 35: 87-129.

105. Sokol DK, Maloney B, Westmark CJ, Lahiri DK (2019) Novel contribution of secreted amyloid- $\beta$ precursor protein to white matter brain enlargement in autism spectrum disorder. Front Psychiatry 10: 165.

106. Guang S, Pang N, Deng X, Yang L, He F, et al. (2018) Synaptopathology Involved in Autism Spectrum Disorder. Front Cell Neurosci 12: 470.

\section{ISSN: 2574-1241}

DOI: 10.26717/BJSTR.2019.19.003321

Castejón 0J. Biomed J Sci \& Tech Res

This work is licensed under Creative

Commons Attribution 4.0 License

Submission Link: https://biomedres.us/submit-manuscript.php
107. Gilbert J, Man HY (2017) Fundamental Elements in Autism: From Neurogenesis and Neurite Growth to Synaptic Plasticity. Front Cell Neurosci 11: 359

108. Pavăl DA (2017) Dopamine hypothesis of autism spectrum disorder Dev Neurosci 39(5): 355-360.

109. Veenstra VanderWeele J, Muller CL, Iwamoto H, Sauer JE, Owens WA, et al. (2012) Autism gene variant causes hyperserotonemia, serotonin receptor hypersensitivity, social impairment and repetitive behavior. Proc Natl Acad Sci U S A 109(14): 5469-5474.

110. Muller CL, Anacker AMJ, Veenstra VanderWeele J (2016) The serotonin system in autism spectrum disorder: from biomarker to animal models. Neuroscience 321: 24-41.

111. Sadek A, Berk LS, Mainess K, Daher NS (2018) A Pilot Study: Parent perceptions of behavior change in their child with autism spectrum disorder following high antioxidant cacao consumption. Integr Med (Encinitas) 17(5): 31-38.

112. Gillberg C, Fernell E, Kočovská E, Minnis H, Bourgeron T, et al. (2017) The role of cholesterol metabolism and various steroid abnormalities in autism spectrum disorders: A hypothesis paper. Autism Res 10(6): 1022-1044

113. Görker I, Tüzün U (2005) Autistic-like findings associated with a urea cycle disorder in a 4-year-old girl. J Psychiatry Neurosci 30(2): 133135.

$\begin{array}{ll}\text { BIOMEDICAL } & \text { Assets of Publishing with us } \\ \text { RESEARCHES } & \text { - Global archiving of articles } \\ \text { - Immediate, unrestricted online access } & \text { - Rigorous Peer Review Process } \\ & \text { - Authors Retain Copyrights }\end{array}$

